Tersteeg, I.M., Koopman, F.S., Stolwijk-Swuste, J.M., Beelen, A., Nollet, F., CARPA STUDY GROUP.A 5-Yqar longitudinal study of fatigue in patients with late-onset sequelae of poliomyelitis. Archives of Physical Medicine and Rehabilitation: 2011, 92(6), 899-904

\begin{tabular}{|l|l|}
\hline Postprint Version & 1.0 \\
\hline Journal website & $\underline{\text { http://www.sciencedirect.com/science/article/pii/S0003999311000475 }}$ \\
\hline Pubmed link & $\underline{\text { http://www.ncbi.nlm.nih.gov/pubmed/21621666 }}$ \\
\hline DOI & $10.1016 /$ j.apmr.2011.01.005 \\
\hline
\end{tabular}

This is a NIVEL certified Post Print, more info at http://www.nivel.eu.

\title{
A 5-Year Longitudinal Study of Fatigue in Patients With Late-Onset Sequelae of Poliomyelitis
}

Irene M. Tersteeg, MD, Fieke S. Koopman, MD, JANneKe M. STOLWIJK-SwÜste, MD, PhD, ANitA BEELEN, PhD, Frans Nollet, MD, PhD, ON BEHALF OF THE CARPA STUDY GROUP

\begin{abstract}
.
Objectives: To study the severity and 5-year course of fatigue in patients with late-onset sequelae of poliomyelitis (LOSP) and to identify physical and psychosocial determinants of fatigue.

Design: Prospective cohort study with 5 measurements over 5 years.

Setting: University hospital.

Participants: Patients with LOSP (N=168); 89\% of the subjects completed the study.

Interventions: Not applicable.

Main Outcome Measures: Fatigue assessed with the Fatigue Severity Scale (FSS). Potential determinants were perceived physical functioning, bodily pain and mental health, extent of paresis, walking capacity, comorbidity, sleeping disorders, coping, and social support. Associations were investigated by multivariable longitudinal analysis using generalized estimating equations.

Results: The mean FSS score \pm SD at baseline was $5.1 \pm 1.4$, which did not change significantly during the 5-year follow-up.

Reduced physical functioning, increased bodily pain, reduced sleep quality, more psychologic distress, and higher taskoriented coping were independently associated with fatigue.

The extent of paresis and walking capacity were strongly associated with physical functioning.

Conclusions: Fatigue is severe and persistent in patients with LOSP due to physical and psychologic factors, which has implications for counseling and treatment. In addition to the commonly applied interventions targeting physical aspects, psychologic interventions are a potential area for reducing fatigue.
\end{abstract}

MANY POLIO SURVIVORS develop postpolio syndrome (PPS), a condition characterized by new neuromuscular symptoms, after many years of stable functioning. The newly developed symptoms can include progressive muscle weakness or increased muscle fatigability, with or without generalized fatigue, diminished functional capacity, cold intolerance, and muscle and joint pain. ${ }^{1}$ Patients with PPS report fatigue as their most frequent and most debilitating complaint, with a reported incidence of $34 \%$ to $87 \%{ }^{2-6}$ Fatigue in PPS is often severe, sometimes interfering with activities of daily life and leading to restrictions in participation in social activities and limitations in work duties. ${ }^{3}$ Greater insight into the factors that contribute to fatigue is needed to design targeted intervention strategies aimed at reducing fatigue in patients with PPS.

Several studies have reported on the physical and psychologic factors that contribute to fatigue. ${ }^{7-10}$ There are positive correlations between fatigue and physical variables, such as disuse and overuse of muscles and 
pain, as well as with psychologic variables, such as depression. ${ }^{4,8,9} \mathrm{~A}$ crosssectional study showed that vitality (or the absence of general fatigue) in PPS is explained mainly by physical functioning, physical fatigue, pain, and body mass index rather than by psychologic factors like mental health and mental fatigue. ${ }^{10}$ Notably, factors that impact fatigue have the potential for providing treatment opportunities. For example, physical factors might be addressed with exercise training and physical therapy aimed at enhancing exercise capacity and physical functioning. For psychologic factors, a cognitive behavioral approach may reduce fatigue symptoms in PPS as in other diseases, such as multiple sclerosis. ${ }^{11}$ The Comorbidity and Aging effects in Rehabilitation Patients on Activities (CARPA) study is a prospective and ongoing follow-up study to investigate the course of functioning in patients with late-onset sequelae of poliomyelitis (LOSP) with specific emphasis on the influence of comorbidity and aging.

${ }^{12,13}$ The term LOSP is used rather than PPS because PPS implies the exclusion of comorbid conditions as causes of muscle weakness and fatigue. ${ }^{1}$ To date, physical, psychologic, and social factor data have been collected from 168 patients over a period of 5 years. The CARPA study provides a unique opportunity to study fatigue in a large sample using a longitudinal study design. The aims of the present study were to [table 1] characterize the severity, course, and physical and psychosocial variables related to fatigue in patients with late-onset sequelae of poliomyelitis (LOSP).

\section{METHODS}

\section{Participants}

The study group, which consisted of 168 subjects with LOSP, is described in detail elsewhere. ${ }^{12}$ The inclusion criteria for the study were as follows: (1) history of poliomyelitis anterior acuta; (2) the presence of residual paresis in at least 1 extremity; (3) at least 1 consultation with a neurologist or specialist in physical medicine and rehabilitation during the previous 5 years for late-onset symptoms; (4) 45 to 85 years of age; and (5) no medical condition indicating a life expectancy of less than 1 year. The study was approved by the medical ethics committees of the 2 participating university hospitals. All participants provided informed written consent. The baseline characteristics of the study group are summarized in table 1.

\section{Assessments}

Five assessments were performed over 5 years: at baseline, and after 1, 2, 3, and 5 years. Each assessment involved completing questionnaires at home and being interviewed and tested at the hospital by a doctor who was not involved in the patient's routine treatment. If patients were not able to visit the outpatient clinic, they were interviewed by phone or visited at home.

\section{Instruments}

The Fatigue Severity Scale (FSS) measures subjective fatigue and is composed of 9 statements that are rated on a scale from 1 (strong disagreement) to 7 (strong agreement). The score is calculated as the mean of the responses to the 9 statements. There is a maximal score of 7, and a score greater than 4 indicates a moderate to high level of fatigue. ${ }^{14}$ The FSS has good internal consistency. ${ }^{15}$ The Medical Outcomes Study 36-Item Short-Form Health Survey (SF-36) is a self-administered health-related quality of life inventory with 36 questions in 8 physical and psychologic domains. ${ }^{16}$ The domain scores range from 0 to 100 , with a higher score indicating higher levels of functioning or wellbeing.

The SF-36 has been translated and validated for the Dutch population. ${ }^{17}$ This study used the following domains: physical functioning, bodily pain, and mental health.

The extent of paresis was assessed with a strength sum-score calculated from manual muscle tests according to the Medical Research Council scale; the strength sum-score includes 16 leg-muscle groups and 10 arm-muscle groups (score range, $0-130$ ) and was described previously by Nollet, ${ }^{6}$ Meldrum, ${ }^{18}$ and colleagues.

Walking capacity was determined by measuring the distance covered during a 2-minute walking test at self-preferred speed on an indoor circuit. This test has excellent test-retest reliability and has been recommended as a capacity qualifier of physical functioning for patients with LOSP. ${ }^{19}$ Comorbidities were assessed with the Cumulative Illness Rating Scale (CIRS). The CIRS rates 13 body systems using a 5-point 
(pathophysiologic) severity scale, with 0 being no disease and 4 being a life-threatening disease. A sum score was calculated as an aggregate measure of comorbidity (comorbidity count). The CIRS is a valid and reliable method of measuring comorbidities that can be used in clinical research. ${ }^{20}$ Sleep disorders were assessed using the Nottingham Health Profile, a self-administered questionnaire that measures health status. ${ }^{21}$ The Nottingham Health Profile domain sleep consists of 5 questions with a score ranging from 0 (no complaints) to 100 (indicating very poor sleep).

Coping was measured using the Coping Inventory for Stressful Situations Short Form, which has 21 questions that are scored using a 5-point scale. The Coping Inventory for Stressful Situations is composed of 3 scales that relate to different styles of coping: task-oriented coping, emotional coping, and avoidance. A higher scale score indicates that the particular coping style is used more often. ${ }^{22}$ Social support was assessed with the questionnaire Social Support Scale (in Dutch), which consists of 12 questions scored on a 5-point scale. The total score is calculated and ranges from 12 (no social support) to 60 (good perceived social support). ${ }^{23}$

\section{Data Analysis}

Demographic data were analyzed with descriptive statistics.

The time course of changes in fatigue during the 5-year study was analyzed with linear generalized estimating equations (GEE), a linear regression analysis that takes into account the dependence of the observations within an individual subject over time and allows inclusion of subjects with missing data.

The correlation structure was set at exchangeable (ie, correlation coefficients between the first and 4 successive assessments are approximately equal). In all regressions, time was modeled as a continuous variable that was expressed in years. The impact of new muscle weakness and/or new muscle fatigue at baseline on the time course of fatigue was tested using the interaction term time by new neuromuscular symptoms. Variables that were potentially associated with fatigue were analyzed with multivariable repeated-measures models. To determine whether a variable should be included, separate univariate models were tested in which only 1 variable was added to the model at a time. When a variable was significantly correlated with fatigue $(P<.01)$, it was included in the final model.

Univariate associations between the potential variables were determined to assess multicollinearity. In case of a correlation of 0.75 or higher, the variable with the lowest correlation with fatigue was omitted. The identified variables were entered into the overall multivariable regression model. GEE analyses were performed using STATA (version 9.0). ${ }^{\mathrm{a}}$

\section{RESULTS}

During the course of the study, 5 patients died (2 patients of unknown causes, 1 patient of lung carcinoma, 1 patient of adrenal gland carcinoma, and 1 patient of complications during treatment for lymphoma). Two patients died after the second year, and 3 died after the third year. At 5 years, $14(8.3 \%)$ patients had been lost for follow-up. Group mean values for fatigue and the physical and psychosocial variables potentially related to fatigue at each assessment are shown in table 2.

\section{[TABLE 2]}

The severity of fatigue at baseline was high. The average score \pm SD on the FSS was $5.1 \pm 1.4$, and $80 \%$ of the patients had FSS fatigue scores greater than 4 . The fatigue scores did not change significantly during the 5 -year follow- up ( $\beta=-.033, \mathrm{SE}=.02, P=.097$ ) (see table 2 ). Patients with new muscle weakness and/or new muscle fatigue had higher fatigue scores $(\beta=1.3, \mathrm{SE}=0.3, P=.001)$, but the course of their fatigue during the 5 -year follow-up did not differ from that of patients without new weakness and/or fatigue.

Over the course of the study, there was a significant decrease in physical functioning (SF-36) and walking capacity and a significant increase in comorbidity and extent of paresis. The most frequently reported comorbidities affected the following body systems: vascular (eg, hypertension) $(26.8 \%$ of the patients at baseline and $32.2 \%$ at last assessment); skeletal, muscular, and skin (eg, arthrosis) $(65.5 \%$ of the patients at baseline and $65.7 \%$ at last assessment); and endocrine and metabolic (eg, diabetes and hypothyroidism) 
(19.1\% of the patients at baseline and $35.6 \%$ at last assessment). The other potential variables did not change significantly over the 5 -year period.

Univariate analysis revealed significant negative associations between fatigue and physical functioning ( $\beta=-$ $.022, P<.001)$, walking capacity $(\beta=-.005, P<.024)$, pain $(\beta=.016, P<.001)$, and mental health $(\beta=-.012$,

$P .001$ ) (table 3 ). Univariate analysis also revealed significant positive associations between fatigue and all 3 coping styles, that is, task-oriented coping ( $\beta=.037, P=.002)$, emotional coping $(\beta=.043, P<.001)$, and avoidance $(\beta=.027, P<.022)$, as well as sleep disorders $(\beta=.007, P<.001)$. There was no significant difference in the severity and course of fatigue in men and women.

Correlations between the variables that were significantly associated with fatigue were all below the cut-off value of 0.75 for multicollinearity. Hence, all variables with a univariate association were entered into the multivariable regression model for fatigue, revealing that physical functioning (standardized $\beta=-.305$, $P<.001$ ), pain (standardized $\beta=-.161, P<.001$ ), task-oriented coping (standardized $\beta=.155, P=.001$ ), mental health (standardized $\beta=.100, P=.008$ ), and sleep disorders (standardized $\beta=.084, P=.023$ ) were all independently associated with fatigue (table 4$)$.

Physical functioning was strongly associated with walking capacity $(\beta=.332, \mathrm{SE}=.035, P .001)$, which, in turn, was negatively associated with the extent of paresis $(\beta=1.026, \mathrm{SE}=.151, P<.001)$

\section{DISCUSSION}

This study demonstrates that fatigue is a common, severe, persistent, and multidimensional complaint in patients with LOSP. Confirming previous reports, fatigue in this study sample was severe, with an average FSS score of 5.1..$^{2-4,6,24}$ Longitudinal studies of fatigue in polio survivors with late symptoms are scarce. In our study, fatigue remained high over a 5-year period. This result contrasts with findings in an earlier study of patients with PPS in which energy and fatigue, which were measured using different questionnaires than those used in this study, had improved significantly after 6 years compared to baseline. ${ }^{6}$ However, that study found a marked improvement during the first year of follow-up that was followed by gradual worsening of fatigue over the next 5 years. In our study sample, fatigue did not change over time, despite the fact that muscle strength and physical function gradually declined. ${ }^{13}$ On the other hand, the regular medical and rehabilitation care that was provided to the patients was not specifically aimed at reducing fatigue.

\section{[TABLE 3]}

We found that fatigue was associated with physical as well as psychosocial variables, namely physical functioning, pain, sleep disorders, mental health, and task-oriented coping. These results are inline with a smaller, cross-sectional study by Trojan et $\mathrm{al}^{7}$ that used different instruments. It is useful to consider how these variables might be connected with perceived fatigue.

Fatigue was associated with perceived restrictions in physical functioning. This could be explained by activities of daily living requiring greater effort of weakened muscles and reduced movement efficiency. ${ }^{10,24}$ Paresis was not a determinant of fatigue per se, but was associated with walking capacity, which in turn was associated with physical functioning. These associations are inline with reported relationships between walking capacity and extent of paresis and between walking capacity and physical functioning. ${ }^{13,25-27}$ This implies that polio survivors with more severe paresis are less capable of walking and perceive their physical functioning to be more demanding and fatiguing. It was interesting that fatigue remained unchanged while perceived physical functioning declined, as this may imply that the patients reduced their physical activity over time to avoid further increases in fatigue. This remains speculative, however, because we did not measure activity, which was found by Trojan ${ }^{7}$ to be significantly associated with fatigue.

A higher level of pain was associated with more fatigue, which is in agreement with earlier cross-sectional studies. ${ }^{7,28}$ Pain is a common and persistent problem in patients with LOSP and may cause people to diminish their activities, leading to deconditioning and hence fatigue. ${ }^{7,28}$ Pain could cause sleep disorders and thus result in more fatigue. However, pain was found to be a variable correlated with fatigue independent of sleep disorders, and this was also reported for fibromyalgia.

${ }^{29}$ Sleep disorders were positively associated with fatigue in our study sample, in agreement with the study by Trojan, ${ }^{7}$ as well as with observations in the general population. ${ }^{30}$ Sleep disorders have been reported 
more frequently by patients with LOSP than control subjects and have been related to a higher prevalence of obstructive sleep apnea syndrome and periodic limb movements in sleep. ${ }^{31-33}$ Lower mental health scores on the SF-36 were associated with fatigue, which is in agreement with other studies of polio survivors, as well as with studies in the general population. ${ }^{4,10,34}$

\section{[TABLE 4]}

Mental health in our cohort was comparable to that in a Dutch reference population, providing corroboration that psychologic problems and psychiatric diagnoses (eg, depression) are not found more frequently in polio survivors with late symptoms. ${ }^{10,35,36} \mathrm{~A}$ task-oriented coping style correlated with higher levels of fatigue. Task-oriented coping, also known as problem-focused coping, consists of actively resolving a problem by acting on the environment or oneself. ${ }^{37}$ Emotional coping is the adaptation of emotional reactions associated with the problem; it is more dominant in situations in which illness-related problems cannot be resolved. ${ }^{37,38}$ As fatigue in patients with LOSP cannot be cured, a higher use of emotional coping is expected. Polio survivors have been found to use problemfocused coping more often than emotional coping. ${ }^{4,38}$ The higher use of task-oriented coping may be due to the strong motivation of patients with LOSP to be self-sufficient, to actively solve problems, and, often, to push themselves beyond their physical abilities to achieve their goals. ${ }^{39,40}$ Thus, patients with LOSP may persist in their behaviors despite decreasing physical functioning at the cost of increased fatigue. Both emotional and task-oriented coping were associated with fatigue in this study. In the multivariable model, however, emotional coping was not an independent factor. That may be because of an overlap in the concepts of emotional coping and mental health.

\section{Study Limitations}

This study also has some limitations. Associations between fatigue and the different variables were investigated with GEE.

However, this technique cannot answer the question of whether relationships are causal. Causality and treatment amenability need to be investigated in future studies.

We studied a limited number of possible variables for fatigue.

For example, we did not include treatments patients received as a variable. During the course of the study, 14 patients $(8.3 \%)$ participated in a multidisciplinary rehabilitation program because of PPS, which may have affected their fatigue.

Comorbidity was quantified using the CIRS with a cumulative count of prevalent comorbid conditions in 13 body systems.

This assumes that all comorbidities have an equal effect on outcome in terms of magnitude and direction and does not take into account the differential effects of disease severity.

This may explain our finding that comorbidity was not associated with fatigue.

Patients who reported new muscle weakness or muscle fatigue at baseline did not show a different course of fatigue than the patients without new muscle weakness or fatigue. This observation is in line with an earlier report from CARPA that the course of disability and muscle strength did not differ between patients with and without new muscle weakness or fatigue. ${ }^{13}$ It is therefore likely that the results of this study are also applicable to patients with PPS.

Despite these limitations the strengths of our study are its longitudinal design, which included 5 assessments over a 5-year period, the large cohort size $(\mathrm{N}=168)$, the wide age range $(45-81 \mathrm{y})$, and the low dropout rate $(7.7 \%$ over $5 y)$.

\section{Clinical Implications}

This study identified potentially modifiable variables associated with fatigue: physical functioning, pain, sleep disorders, mental health, and task-oriented coping. These variables could provide opportunities for treatment. Fatigue in polio survivors with LOSP is a multidimensional problem, and treatment should be multidisciplinary with attention to both physical and psychologic determinants, including pain management, exercise therapy, and cognitive behavioral therapy. Although not tested in patients with LOSP, the effectiveness of cognitive behavioral therapy in reducing fatigue has been demonstrated in 
Tersteeg, I.M., Koopman, F.S., Stolwijk-Swuste, J.M., Beelen, A., Nollet, F., CARPA STUDY GROUP.A 5-Yqar longitudinal study of fatigue in patients with late-onset sequelae of poliomyelitis. Archives of Physical Medicine and Rehabilitation: 2011, 92(6), 899-904

chronic fatigue syndrome, fatigued cancer survivors, and multiple sclerosis. ${ }^{11,41,42}$ A recent pilot study on the effectiveness of a multidisciplinary rehabilitation program for PPS showed prolonged benefits, such as decreased fatigue. ${ }^{43}$ More research on multidisciplinary treatment protocols targeting the variables associated with fatigue is still needed. The efficacy of 2 interventions on fatigue (ie, physical therapy and cognitive behavioral therapy) is currently being evaluated in an ongoing multicenter randomized trial in PPS patients. ${ }^{44}$

\section{CONCLUSIONS}

Fatigue in patients with LOSP is a severe and persistent problem that correlates with physical and psychologic variables.

Some of these variables can potentially be modified, which may provide an opportunity for treating fatigue. Further study is needed to investigate the efficacy of treatment directed at modifying these physical and psychologic determinants.

Acknowledgements: The following participated in the CARPA study group: J.M. Stolwijk-Swüste, MD, $\mathrm{PhD}$, I.M. Tersteeg, MD, A. Beelen, PhD, F. Nollet, MD, PhD, G.J. Lankhorst, MD, PhD, J. Dekker, PhD, G.M. van Dijk, PhD, B. Post, MD, PhD, R.J. de Haan, PhD, Cindy Veenhof, PhD, and H. Speelman, MD, $\mathrm{PhD}$.

\section{REFERENCES}

1. March of Dimes Foundation. Post-polio syndrome: identifying best practices in diagnosis \& care. White Plains: March of Dimes; 2001.

2. Schanke AK, Stanghelle JK. Fatigue in polio survivors. Spinal Cord 2001;39:243-51.

3. Packer TL, Sauriol A, Brouwer B. Fatigue secondary to chronic illness: postpolio syndrome, chronic fatigue syndrome, and multiple sclerosis. Arch Phys Med Rehabil 1994;75:1122-6.

4. Schanke AK. Psychological distress, social support and coping behaviour among polio survivors: a 5year perspective on 63 polio patients. Disabil Rehabil 1997;19:108-16.

5. Ramlow J, Alexander M, LaPorte R, Kaufmann C, Kuller L. Epidemiology of the post-polio syndrome. Am J Epidemiol 1992; 136:769-86.

6. Nollet F, Beelen A, Twisk JW, Lankhorst GJ, De Visser M. Perceived health and physical functioning in postpoliomyelitis syndrome: a 6-year prospective follow-up study. Arch Phys Med Rehabil 2003;84:104856.

7. Trojan DA, Arnold DL, Shapiro S, et al. Fatigue in postpoliomyelitis syndrome: association with disease related, behavioral, and psychosocial factors. PM R 2009;1:442-9.

8. Agre JC, Rodriquez AA, Tafel JA. Late effects of polio: critical review of the literature on neuromuscular function. Arch Phys Med Rehabil 1991;72:923-31.

9. Vasiliadis HM, Collet JP, Shapiro S, Venturini A, Trojan DA. Predictive factors and correlates for pain in postpoliomyelitis syndrome patients. Arch Phys Med Rehabil 2002;83:1109-15.

10. Ostlund G, Wahlin A, Sunnerhagen KS, Borg K. Vitality among Swedish patients with post-polio: a physiological phenomenon. J Rehabil Med 2008;40:709-14.

11. van Kessel K, Moss-Morris R, Willoughby E, Chalder T, Johnson MH, Robinson E. A randomized controlled trial of cognitive behavior for multiple sclerosis fatigue. Psychosom Med 2008;70: 205-13.

12. Stolwijk-Swüste JM, Beelen A, Lankhorst G, Nollet F; CARPA study group. Impact of age and comorbidity on the functioning of patients with sequelae of poliomyelitis: a cross-sectional study. J Rehabil Med 2007;39:59-62.

13. Stolwijk-Swüste JM, Tersteeg I, Beelen A, Lankhorst GJ, Nollet F; CARPA Study Group. The impact of age and comorbidity on the progression of disability in late-onset sequelae of poliomyelitis. Arch Phys Med Rehabil 2010;91:523-8.

14. Valko PO, Bassetti CL, Bloch KE, Held U, Baumann CR. Validation of the fatigue severity scale in a Swiss cohort. Sleep 2008;31:1601-7.

15. Horemans HL, Nollet F, Beelen A, Lankhorst GJ. A comparison of 4 questionnaires to measure fatigue in postpoliomyelitis syndrome. Arch Phys Med Rehabil 2004;85:392-8.

16. McHorney CA, Ware JE Jr, Raczek AE. The MOS 36-item Short-Form Health Survey (SF-36): II. Psychometric and clinical tests of validity in measuring physical and mental health constructs. Med Care 1993;31:247-63. 
Tersteeg, I.M., Koopman, F.S., Stolwijk-Swuste, J.M., Beelen, A., Nollet, F., CARPA STUDY GROUP.A 5-Yqar longitudinal study of fatigue in patients with late-onset sequelae of poliomyelitis. Archives of Physical Medicine and Rehabilitation: 2011, 92(6), 899-904

17. Aaronson NK, Muller M, Cohen PD, et al. Translation, validation and norming of the Dutch language version of the SF-36 Health survey in community and chronic disease populations. J Clin Epidemiol 1998;51:1055-68.

18. Meldrum D, Cahalane E, Conroy R, Guthrie R, Hardiman O. Quantative assessment of motor fatigue: normative values and comparison with prior-polio patients. Amyotroph Lateral Scler 2007;8:170-6.

19. Stolwijk-Swüste JM, Beelen A, Lankhorst GJ, Nollet F; CARPA study group. SF36 physical functioning scale and 2-minute walk test advocated as core qualifiers to evaluate physical functioning in patients with late-onset sequelae of poliomyelitis. J Rehabil Med 2008;40:387-92.

20. De Groot V, Beckerman H, Lankhorst GJ, Bouter LM. How to measure comorbidity: a critical review of available methods. J Clin Epidemiol 2003;56:221-9.

21. Erdman RA, Passchier J, Kooijman M, Stronks DL. The Dutch version of the Nottingham Health Profile: investigations of psychometric aspects. Psychol Rep 1993;72:1027-35.

TABLES

\section{List of Abbreviations}

\begin{tabular}{ll}
\hline CARPA & $\begin{array}{c}\text { Comorbidity and Aging effects in Rehabilitation } \\
\text { Patients on Activities }\end{array}$ \\
CIRS & Cumulative Illness Rating Scale \\
FSS & Fatigue Severity Scale \\
GEE & generalized estimating equations \\
LOSP & late-onset sequelae of poliomyelitis \\
PPS & postpolio syndrome \\
SF-36 & Medical Outcomes Study 36-Item Short-Form \\
& Health Survey
\end{tabular}

Table 1: Patient Characteristics

\begin{tabular}{lc}
\hline \multicolumn{1}{c}{ Characteristics at Baseline } & $(\mathrm{N}=168)$ \\
\hline Sex (men/women) & $67 / 101$ \\
Age, y (mean $\pm \mathrm{SD}$ ) & $59 \pm 8$ \\
Range, y & $45-81$ \\
Marital status ( $\mathrm{n}$ married [\%]) & $121(72)$ \\
Time since onset of polio, y (mean $\pm \mathrm{SD})$ & $55 \pm 8$ \\
Body parts affected by polio (n [\%]) & \\
Right arm & $28(17)$ \\
Left arm & $31(39)$ \\
Back and abdomen & $22(13)$ \\
Right leg & $106(63)$ \\
Left leg & $107(64)$ \\
New neuromuscular symptoms (n [\%]) & $147(88)$ \\
New muscle weakness (n [\%]) & $138(82)$ \\
New muscle fatigue (n [\%]) & $114(68)$ \\
\hline
\end{tabular}


Tersteeg, I.M., Koopman, F.S., Stolwijk-Swuste, J.M., Beelen, A., Nollet, F., CARPA STUDY GROUP. A 5-Yqar longitudinal study of fatigue in patients with late-onset sequelae of poliomyelitis. Archives of Physical Medicine and Rehabilitation: 2011, 92(6), 899-904

Table 2: Descriptive Data for Independent and Dependent Variables

\begin{tabular}{|c|c|c|c|c|c|c|}
\hline $\begin{array}{c}\text { Characteristics Fatigue and Potential } \\
\text { Determinants }\end{array}$ & Baseline & T1 & T2 & T3 & T4 & Course $\beta$ (SE) \\
\hline Fatigue (FSS) (1-7) & $5.1 \pm 1.4(167)$ & $5.2 \pm 1.4(160)$ & $5.2 \pm 1.4(160)$ & $5.1 \pm 1.4(156)$ & $5.0 \pm 1.5(148)$ & $\begin{array}{l}.033(.020) \\
P=.097\end{array}$ \\
\hline SF-36 physical functioning $(0-100)$ & $39 \pm 24(167)$ & $38 \pm 24$ (159) & $37 \pm 24(155)$ & $35 \pm 24$ (149) & $36 \pm 25(149)$ & $\begin{array}{c}-0.689(0.262) \\
P=.009\end{array}$ \\
\hline SF-36 bodily pain (0-100) & $56 \pm 22(168)$ & $58 \pm 24(162)$ & $58 \pm 23(160)$ & $56 \pm 24(158)$ & $56 \pm 22(149)$ & $\begin{array}{c}-.264(.322) \\
P=.413\end{array}$ \\
\hline Extent of paresis* $(0-130)$ & $109 \pm 20(168)$ & $109 \pm 18(157)$ & $109 \pm 17(154)$ & $107 \pm 20(147)$ & $106 \pm 17(149)$ & $\begin{array}{c}-.722(.081) \\
P<.001\end{array}$ \\
\hline Walking capacity (m) & $126 \pm 34(152)$ & $124 \pm 33(144)$ & $127 \pm 34(139)$ & $127 \pm 34(131)$ & $124 \pm 33(113)$ & $\begin{array}{c}-.864(.042) \\
P=.008\end{array}$ \\
\hline Comorbidity (CIRS total score) (0-52) & $6.5 \pm 3.7(168)$ & $6.9 \pm 3.9(158)$ & $7.0 \pm 3.8(157)$ & $7.7 \pm 4.0(155)$ & $8.1 \pm 4.2(149)$ & $\begin{array}{l}.299(.42) \\
P<.001\end{array}$ \\
\hline Sleep disorders (NHP sleep) $(0-100)$ & $22 \pm 30(168)$ & $24 \pm 29(160)$ & $24 \pm 30(162)$ & $25 \pm 30(153)$ & $25 \pm 29(149)$ & $\begin{array}{l}.442(.408) \\
P=.279\end{array}$ \\
\hline SF-36 mental health $(0-100)$ & $75 \pm 17(168)$ & $72 \pm 19(161)$ & $75 \pm 18(160)$ & $75 \pm 17(155)$ & $76 \pm 19(149)$ & $\begin{array}{l}.315(.262) \\
P=.229\end{array}$ \\
\hline Task-oriented coping style (0-35) & $25 \pm 6(167)$ & $25 \pm 6(160)$ & $24 \pm 7(161)$ & $25 \pm 6(158)$ & $25 \pm 6(147)$ & $\begin{array}{l}.079(.088) \\
P=.372\end{array}$ \\
\hline Emotional coping style (0-35) & $17 \pm 6(167)$ & $17 \pm 6(160)$ & $16 \pm 6(161)$ & $17 \pm 6(158)$ & $16 \pm 6(147)$ & $\begin{array}{c}-.0150(.080) \\
P=.06\end{array}$ \\
\hline Avoidance coping style (0-35) & $14 \pm 6(167)$ & $15 \pm 6(160)$ & $15 \pm 6(161)$ & $15 \pm 6(158)$ & $14 \pm 6(147)$ & $\begin{array}{c}-.043(.087) \\
P=.622\end{array}$ \\
\hline Social support (0-60) & $55 \pm 8(166)$ & $56 \pm 8(160)$ & $56 \pm 8(162)$ & $55 \pm 9(156)$ & $56 \pm 7(149)$ & $\begin{array}{c}.191(.098) \\
P=.051\end{array}$ \\
\hline
\end{tabular}

NOTE. Values are mean $+\mathrm{SD}(\mathrm{n})$ or as otherwise noted.

Abbreviations: NHP, Nottingham Health Profile; T1, 1-year follow-up; T2, 2-year follow-up; T3, 3-year follow-up; T4, 5-year follow-up.

*Extent of paresis: score range 0 to 130 with 130 as full muscle capacity in all of the 26 muscle groups.

Table 3: Correlations Among All Dependent and Independent Variables

\begin{tabular}{|c|c|c|c|c|c|c|c|c|c|c|c|c|}
\hline Variables & 1 & 2 & 3 & 4 & 5 & 6 & 7 & 8 & 9 & 10 & 11 & 12 \\
\hline 1. Fatigue & 1 & & & & & & & & & & & \\
\hline 2. Physical functioning & $-.40^{\dagger}$ & 1 & & & & & & & & & & \\
\hline 3. Pain & $-.41^{\dagger}$ & $.33^{\dagger}$ & 1 & & & & & & & & & \\
\hline 4. Extent of paresis & -.09 & $.49^{+}$ & $.19^{*}$ & 1 & & & & & & & & \\
\hline 5. Walking capacity & $-.15^{*}$ & $.69^{+}$ & $.28^{*}$ & $.62^{+}$ & 1 & & & & & & & \\
\hline 6. Comorbidity & .12 & $-.29^{\dagger}$ & $-.28^{\dagger}$ & $-.06^{\dagger}$ & $-.26^{\dagger}$ & 1 & & & & & & \\
\hline 7. Sleep disorders & $.19^{\dagger}$ & -.10 & $-.24^{+}$ & -.04 & -.09 & $.22^{*}$ & 1 & & & & & \\
\hline 8. Mental health & $-.26^{\dagger}$ & .04 & $.25^{+}$ & -.11 & .04 & -.13 & $-.24^{\dagger}$ & 1 & & & & \\
\hline 9. Task-oriented coping & $.19^{+}$ & .06 & -.07 & -.03 & .08 & -.11 & -.05 & -.04 & 1 & & & \\
\hline 10. Emotional coping & $.28^{\dagger}$ & -.10 & $-.23^{\dagger}$ & -.05 & -.08 & .11 & $.20^{\dagger}$ & $-.46^{\dagger}$ & $.19^{\dagger}$ & 1 & & \\
\hline 11. Avoidance coping & $.09^{*}$ & -.06 & -.07 & -.05 & -.07 & -.11 & .08 & -.10 & $.25^{\dagger}$ & $.25^{\dagger}$ & 1 & \\
\hline 12. Social support & .11 & .11 & -.05 & -.02 & -.05 & -.10 & -.17 & $.28^{\dagger}$ & .00 & $.21^{\dagger}$ & $.18^{*}$ & 1 \\
\hline
\end{tabular}

Table 4: Linear Longitudinal Multivariable Model

\begin{tabular}{lcr}
\hline \multicolumn{1}{c}{ Determinant } & Standardized $\beta$ & $P$ \\
\hline Physical functioning & -0.305 & $<.001$ \\
Pain & -0.161 & $<.001$ \\
Task-oriented coping & 0.155 & $<.001$ \\
Mental health & 0.100 & .008 \\
Sleep disorders & 0.084 & .023 \\
\hline
\end{tabular}

